\title{
DUTH at SemEval-2017 Task 5: Sentiment Predictability in Financial Microblogging and News Articles
}

\author{
Symeon Symeonidis \\ John Kordonis \\ Dimitrios Effrosynidis \\ Database \& Information Retrieval research unit, \\ Department of Electrical \& Computer Engineering, \\ Democritus University of Thrace, Xanthi 67100, Greece \\ \{ssymeoni, ioankord1, dimievfr, avi\}@ee.duth.gr
}

Avi Arampatzis

\begin{abstract}
We present the system developed by the team DUTH for the participation in Semeval-2017 task 5 - Fine-Grained Sentiment Analysis on Financial Microblogs and News, in subtasks A and B. Our approach to determine the sentiment of Microblog Messages and News Statements \& Headlines is based on linguistic preprocessing, feature engineering, and supervised machine learning techniques. To train our model, we used Neural Network Regression, Linear Regression, Boosted Decision Tree Regression and Decision Forrest Regression classifiers to forecast sentiment scores. At the end, we present an error measure, so as to improve the performance about forecasting methods of the system.
\end{abstract}

\section{Introduction}

Social media sentiment is an important indicator of public opinion. Determining sentiment can be valuable in a number of applications including brand awareness, product launches, and detecting political trends. Many microblogging platforms such as Twitter and StockTwits have become very popular and are employed by many traders and investors. Recently, many studies (Piñeiro-Chousa et al., 2016; Van de Kauter et al., 2015; Kordonis et al., 2016) used sentiment from social media and financial news articles trying to analyze market movements.

This paper describes our submissions to SemEval 2017 task 5 (Cortis et al., 2017), which deals with sentiment analysis in microblog messages for SubTask A, and sentences for news headlines for SubTask B. In SubTask A, our model was ranked last because of a submission format error. We perform error measures in order to obtain a better understanding of the strengths of these particularly new tasks and to improve the performance about forecasting methods of our model (Armstrong and Collopy, 1992). For Subtask B, our team was ranked 24th from 29 teams.

The rest of this paper is structured as follows: Section 2 provides our system's description. Section 3 presents the experiments and some unofficial results used in analyzing the system's performance. Finally, conclusions and further directions for research are presented in Section 4.

\section{System Description}

In this section we present the details of our sentiment analysis system, feature extraction and some statistics about preprocessing.

\subsection{Dataset}

The Task 5 organizers (Cortis et al., 2017) provided a training and testing set for both subtasks. For subtask A, resources of Microblog messages were Stocktwits and Twitter, which have been annotated for fine-grained sentiment ${ }^{1}$. A collection of financially relevant news headlines which have been annotated for fine-grained sentiment, from sources such as Yahoo Finance, are given from Task Organizers for subtask B ${ }^{2}$. Some statistics about the datasets are presented in table 1 .

\subsection{Pre-processing and Feature Engineering}

To make it suitable for reliable analysis, the data had to be pre-processed. Moreover, feature hashing was used as an approach according to (Da Silva et al., 2014), to reduce the number of features provided as input from pre-processing to

\footnotetext{
${ }^{1}$ https://bitbucket.org/ssix-project/semeval-2017-task-5subtask-1/

${ }^{2}$ https://bitbucket.org/ssix-project/semeval-2017-task-5subtask-2/
} 


\begin{tabular}{l|c|c}
\hline & train & test \\
\hline Task A & 1534 & 799 \\
Task B & 1142 & 491 \\
\hline
\end{tabular}

Table 1: Number of tweets in training (train) and testing (test) data for subtask A and B.

a learning algorithm. As first step of our approach we chose to normalize the tweet text by performing the following operations:

- Remove numbers

- Remove punctuation

- Replace all user mentions and URL addresses, which were normalized to “@user" and "URL"

\section{- Convert to lower case}

Furthermore, we chose to compute the counts and cumulative frequencies of the words in the tweets. The NLTK (Bird et al., 2009) package contains two tools to help:

- The regexp_tokenize function tokenizes the text. Tokenization is the process of dividing the text into its component tokens. In this case, the tokens are all words, since we are working with normalized text.

- The FreqDist function computes the frequency distribution of words in a text corpus. A Python Pandas data frame (McKinney, 2010) is then computed from the word frequency array.

The most frequent words are in the head of the new data frame. Of these 20 most frequent words none are likely to give much information on sentiment.

In addition, we implemented a method in order to create a bar plot of word frequency for the 60 most common words, as presented in Figure 1, to comprehend the vocabulary of microblogging messages and news headlines. Unfortunately, we saw that many of the most frequent words are stopwords, such as 'the', 'and', and 'you', which are not likely to be helpful in determining sentiment.

Another tool for examining the frequency of words in a corpus of documents is the cumulative distribution frequency (CDF) plot, as presented in Figure 2.
Figure 1: Frequencies of the most common words

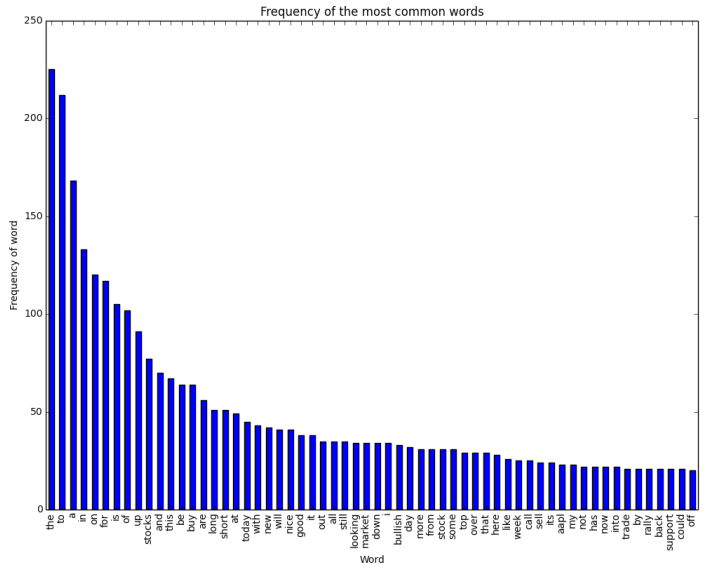

Figure 2: Cummulative fraction of total words vs. words

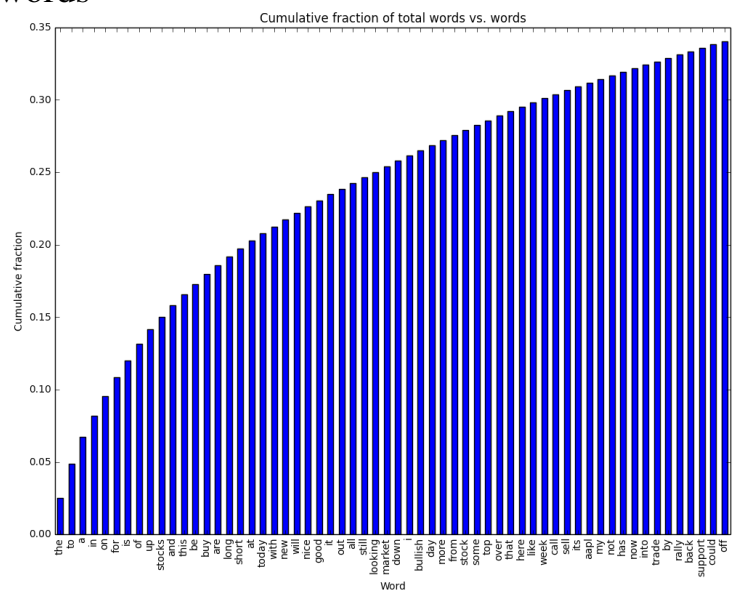

These frequent words, which are largely extraneous, are known as stopwords and should be removed from the text before further analysis, unlike with few studies which take stopwords as features (Mohammad et al., 2013). So, we implemented a method to remove the stopwords from each tweet using nested list comprehensions and execute the code from previous implementation in order to visualize the word frequency.

As before, Figure 3 shows a number of frequent words which are likely to convey sentiment. However, note that these 60 most frequent words only make up about $17 \%$ of the total words, where used in feature extraction. 
Figure 3: Frequencies of the most common words after preprocessing

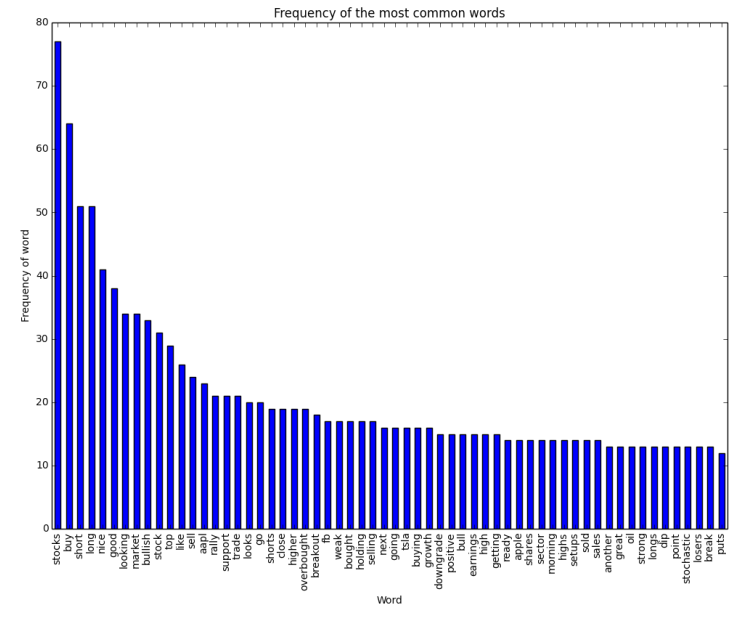

Figure 4: Wordcloud of frequent words after preprocessing

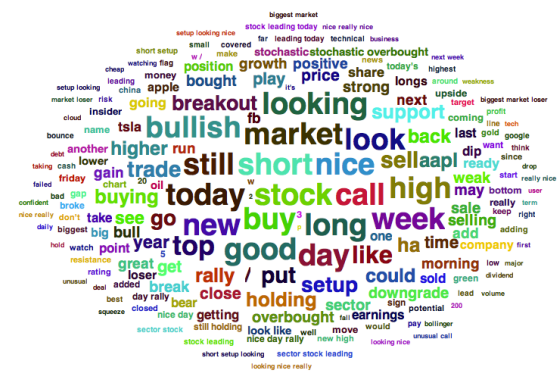

Figure 4 shows the most frequent words on dataset as a wordcloud and helps the researcher to understand if something went wrong in preprocessing.

Now, that we have cleaned the tweet text and removed stopwords, there is one last data preparation step required, stemming the words. Stemming is a process of reducing words to their stems or roots, reduce the vocabulary size and manage the case of data sparseness (Lin and He, 2009). For example, conjugated verbs such as 'goes', 'going', and 'gone' are stemmed to the word "go". Depending on suitable choice, the results can be more or less suitable for the application. In our case, we used the so popular Porter stemmer implemented by the PorterStemmer function in the nltk.stem.porter library.

In subsection 2.2, we described the preprocessing techniques and the features that were extracted for training our model. Section 3 presents the machine learning techniques and algorithms which were used for our experiments.

\section{Experiments}

In this section we present the main methodology implemented in our system for the SemEval 2017 Task 5. In order ti select the feature sets to use for each classifier, we have carried out a number of experiments.

\subsection{Evaluation Metrics}

The evaluation metric used by the task organizers was the cosine similarity, specifically, the metric of Ghosh et al. (2015). Sentiment scores had to be in scale between -1 and 1 . The degree of agreement between predicted values and values from gold labels calculate the final result. In Section 4 the error metrics about our model are presented which are the following:

- Mean Absolute Error

- Root Mean Squared Error

- Relative Absolute Error

- Relative Squared Error

- Coefficient of Determination

The selection of error measures to calibrate our model are based on other related studies (Hippert et al., 2001; Armstrong and Collopy, 1992).

\subsection{Machine Learning Methods}

All system implementation was done using Python and the open-source machine learning toolkit scikit-learn (Pedregosa et al., 2011). In our system we implemented four classification techniques as follows:

- Linear Regression, which attemps to model the relationship between two variables by fitting a linear equation to the training data.

- Boosted Decision Tree Regression, which uses boosting to create an ensemble of regression trees. Boosting aims to learn any tree by fitting the continuing of the trees that preceded ands depends on prior trees. As a result, boosting in a decision tree ensemble contributes to small risk accruracy.

- Decision Forrest Regression, which is a model using an ensemble of decision trees. 
- Neural Network Regression. Although neural networks are known for use in deep learning problems like recognition in images, and for regression problems they are adapted too. So, where a more traditional regression model is not fitting to a solution, neural network regression is suited to these problems.

The above four techniques were chosen empirically and based on related studies (Mittal and Goel, 2012; Ghiassi et al., 2013).

\section{Results}

Below, error measures of our model was done to improve our system's performance about forecasting methods of the system for subtask A. Figure 5 and 6 present the results for Mean Absolute Error, Root mean Squared Error, Relative Absolute Error, Relative Squared Error, and Coefficient of Determination. These metrics represent the performance of our system, without considering the metrics of Task. The results of error measures are promising for accuracy of our model and the prices of errors are not big to have disproportionate impacts for forecasting. According to Task Organizers results, our team got cosine score 0.5879725192 for Subtask B and 0.003076891426 for Subtask A (because of a submission format error).

Figure 5: Linear Regression and Boosted Decision Tree Regression error metrics

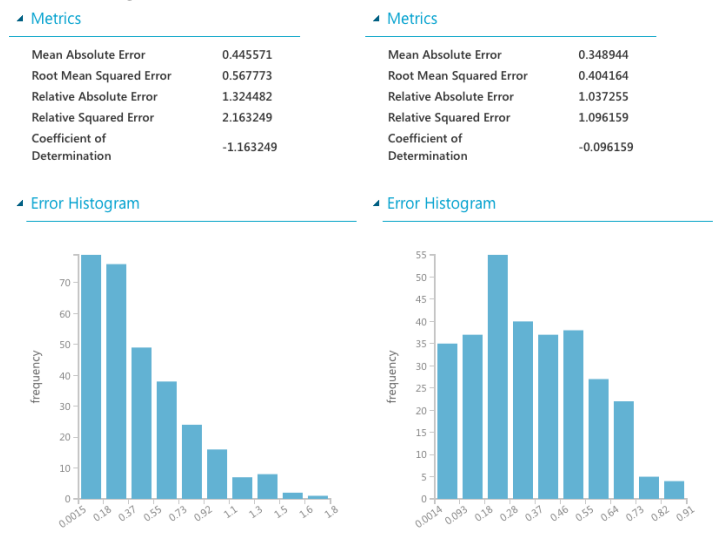

\section{Conclusions}

We presented a supervised regression sentiment analysis system to detect the semantic interpretation of financial texts. Given the above error analysis results, we conclude that our methods for sentiment analysis on financial microblogs and news,
Figure 6: Decision Forrest Regression and Neural Network Regression error metrics
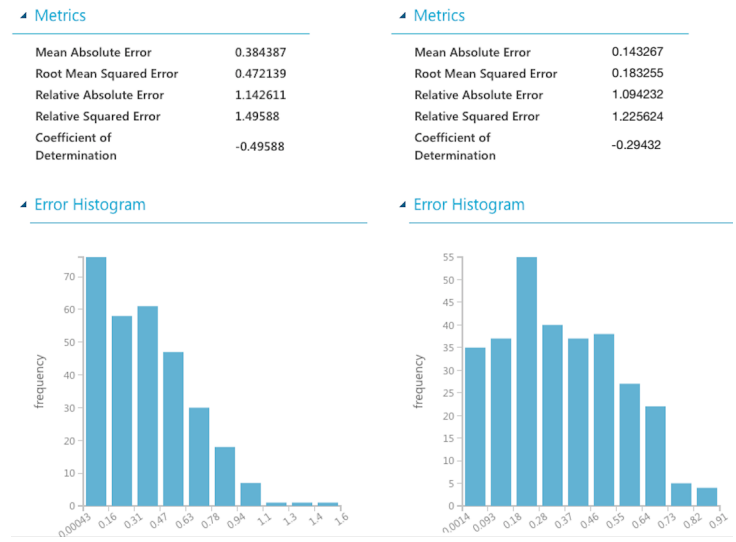

are promising. Future work will focus on feature selection and usage of some lexicons resources to achieve better results.

\section{References}

J. Scott Armstrong and Fred Collopy. 1992. Error measures for generalizing about forecasting methods: Empirical comparisons. International Journal of Forecasting 8(1):69-80. https://doi.org/10.1016/0169-2070(92)90008-W.

Steven Bird, Ewan Klein, and Edward Loper. 2009. Natural Language Processing with Python. O'Reilly Media, Inc., 1st edition.

Keith Cortis, André Freitas, Tobias Dauert, Manuela Huerlimann, Manel Zarrouk, Siegfried Handschuh, and Brian Davis. 2017. Semeval-2017 task 5: Fine-grained sentiment analysis on financial microblogs and news. In Proceedings of the 11th International Workshop on Semantic Evaluation (SemEval-2017). Association for Computational Linguistics, Vancouver, Canada, pages 517-533. http://www.aclweb.org/anthology/S17-2089.

Nadia FF Da Silva, Eduardo R Hruschka, and Estevam R Hruschka. 2014. Tweet sentiment analysis with classifier ensembles. Decision Support Systems 66:170-179.

Manoochehr Ghiassi, James Skinner, and David Zimbra. 2013. Twitter brand sentiment analysis: A hybrid system using n-gram analysis and dynamic artificial neural network. Expert Systems with applications 40(16):6266-6282.

Aniruddha Ghosh, Guofu Li, Tony Veale, Paolo Rosso, Ekaterina Shutova, John Barnden, and Antonio Reyes. 2015. Semeval-2015 task 11: Sentiment analysis of figurative language in twitter. In Proceedings of the 9th International Workshop on Semantic Evaluation (SemEval 2015). Association for Computational Linguistics, Denver, Colorado, pages 
470-478. http://www.aclweb.org/anthology/S152080.

Henrique Steinherz Hippert, Carlos Eduardo Pedreira, and Reinaldo Castro Souza. 2001. Neural networks for short-term load forecasting: A review and evaluation. IEEE Transactions on power systems 16(1):44-55.

John Kordonis, Symeon Symeonidis, and Avi Arampatzis. 2016. Stock price forecasting via sentiment analysis on twitter. In Proceedings of the 20th Pan-Hellenic Conference on Informatics. ACM, New York, NY, USA, PCI '16, pages 36:136:6. https://doi.org/10.1145/3003733.3003787.

Chenghua Lin and Yulan He. 2009. Joint sentiment/topic model for sentiment analysis. In Proceedings of the 18th ACM conference on Information and knowledge management. ACM, pages 375384.

Wes McKinney. 2010. Data structures for statistical computing in python. In Stéfan van der Walt and Jarrod Millman, editors, Proceedings of the 9th Python in Science Conference. pages $51-56$.

Anshul Mittal and Arpit Goel. 2012. Stock Prediction Using Twitter Sentiment Analysis. Tomx.Inf.Elte.Hu (June).

Saif M Mohammad, Svetlana Kiritchenko, and Xiaodan Zhu. 2013. Nrc-canada: Building the stateof-the-art in sentiment analysis of tweets. arXiv preprint arXiv: 1308.6242 .

Fabian Pedregosa, Gaël Varoquaux, Alexandre Gramfort, Vincent Michel, Bertrand Thirion, Olivier Grisel, Mathieu Blondel, Peter Prettenhofer, Ron Weiss, Vincent Dubourg, Jake Vanderplas, Alexandre Passos, David Cournapeau, Matthieu Brucher, Matthieu Perrot, and Édouard Duchesnay. 2011. Scikit-learn: Machine Learning in Python. J. Mach. Learn. Res. 12:2825-2830. http://dl.acm.org/citation.cfm?id=1953048.2078195.

Juan Ramón Piñeiro-Chousa, M Ángeles LópezCabarcos, and Ada María Pérez-Pico. 2016. Examining the influence of stock market variables on microblogging sentiment. Journal of Business Research 69(6):2087-2092.

Marjan Van de Kauter, Diane Breesch, and Véronique Hoste. 2015. Fine-grained analysis of explicit and implicit sentiment in financial news articles. Expert Syst. Appl. 42(11):4999-5010. https://doi.org/10.1016/j.eswa.2015.02.007. 